\title{
Influence of metabolic and other clinicopathologic factors on the prognosis of patients with hepatocellular carcinoma undergoing hepatic resection
}

\author{
Kui Sun Choi, Dong-Sik Kim, Sung Won Jung, Young-Dong Yu, and Sung Ock Suh \\ Department of Surgery, Korea University College of Medicine, Seoul, Korea
}

\begin{abstract}
Backgrounds/Aims: The prognosis of hepatic resection for hepatocellular carcinoma (HCC) in patients with cirrhosis is worse than in those without cirrhosis. In Korea, the hepatitis B virus prevalence rate is higher than in other countries. Therefore, we investigated patients' clinicopathologic and metabolic factors that affect the postoperative outcomes of hepatic resection for HCC in our hospital in Korea. Methods: From August 2000 to December 2012, 171 HCC patients underwent hepatic resections at our institution. Two operative mortality cases and two short-term follow up cases were excluded. Data was collected from a retrospective chart review. There were 133 males (79.6\%) and 34 females $(20.3 \%)$, with a mean age of $58.2 \pm 10.2$ years (range, 22-81 years), and the relationship between clinicopathologic and metabolic factors and the prognosis of patients with HCC undergoing hepatic resection were evaluated by univariate and multivariate analysis. Results: Hypertension, major surgery, perioperative transfusion, resection with radiofrequency ablation (RFA) or cryoablation, and resection margin were risk factors for overall survival, and hypertension, albumin, resection with RFA or cryoablation, perioperative transfusion, and tumor size were risk factors for disease-free survival. Conclusions: We found that hypertension, perioperative transfusion, and resection with RFA or cryoablation were risk factors for both disease-free and overall survival after hepatic resection in HCC patients. Further study is required to clarify the influence of metabolic and other clinicopathologic factors on the prognosis of HCC. (Korean J Hepatobiliary Pancreat Surg 2014;18:105-111)
\end{abstract}

Key Words: Hypertension; Hepatocellular carcinoma; Hepatic resection; Prognosis

\section{INTRODUCTION}

The liver is important in the metabolic processes of the body, and hepatocellular carcinoma (HCC) is the most common primary liver cancer. Radiofrequency ablation (RFA), hepatic resection, and liver transplantation are performed for curative treatment of HCC. ${ }^{1}$ Hepatic resection is still the most common treatment for HCC patients, and there have been many studies about the clinicopathologic factors that affect the prognoses of HCC patients undergoing hepatic resection. However, the hepatitis B virus (HBV) prevalence rate is higher in Korea than in other countries, and patients with HBV have a high incidence of liver cirrhosis. Therefore, it is important to determine the risk factors in order to estimate the prognosis of HCC patients undergoing hepatic resection in Korea.
Metabolic syndrome is a constellation of problems that include insulin resistance, obesity, dyslipidemia, and hypertension. ${ }^{2}$ Obesity and metabolic syndrome are associated with both an increased risk of, and worsened outcomes for, many types of cancer. ${ }^{3}$ In the liver, inflammatory and angiogenic changes due to of underlying insulin resistance and fatty liver disease likely will lead to an increase in the number of patients with HCC in the near future. ${ }^{3}$ Also, hypertension is a risk factor for poor prognoses after resection of hepatitis $\mathrm{C}$ virus infection (HCV)-associated $\mathrm{HCC}^{4}$

The clinicopathologic factors that affect the prognosis of HCC patients undergoing hepatic resection are transfusion, prothrombin time, age, glucose, diabetes, transcatheter arterial embolization, the multifocality of the tumor, the tumor's capsule, tumor staging, infiltration of the

Received: September 11, 2014; Revised: October 16, 2014; Accepted: October 23, 2014

Corresponding author: Dong-Sik Kim

Department of Surgery, Anam Hospital, Korea University Medical Center, 73, Inchon-ro, Seoungbuk-gu, Seoul 136-705, Korea Tel: +82-2-920-6620, Fax: +82-2-921-6620, E-mail: kimds1@korea.ac.kr

Copyright (C) 2014 by The Korean Association of Hepato-Biliary-Pancreatic Surgery

This is an Open Access article distributed under the terms of the Creative Commons Attribution Non-Commercial License (http://creativecommons.org/ licenses/by-nc/3.0) which permits unrestricted non-commercial use, distribution, and reproduction in any medium, provided the original work is properly cited. Korean Journal of Hepato-Biliary-Pancreatic Surgery • pISSN: $1738-6349 \cdot$ eISSN: $2288-9213$ 
cut margin, vascular invasion, lymph nodal infiltration, serum albumin, tumor differentiation, and operation time. ${ }^{5-8}$ With this background, we questioned the relationship between the clinicopathologic factors like hepatitis, diabetes, and hypertension, and the prognosis of HCC patients undergoing hepatic resection in our hospital. We investigated the influence of clinicopathologic and metabolic factors on the outcome of patients with HCC undergoing hepatic resection in our hospital from August 2000 to December 2012.

\section{MATERIALS AND METHODS}

\section{Patient characteristics and evaluation}

A retrospective analysis was done with 167 HCC patients who underwent hepatic resections from August 2000 to December 2012, at the Department of Surgery, Anam hospital, Korea University Medical Center, Seoul, Korea. The last follow-up day was set as the end of December 2012.

All of the patients underwent preoperative clinical evaluation, imaging studies (e.g. chest $\mathrm{x}$-ray and abdominal computed tomography), electrocardiography, and laboratory studies. We could not evaluate complications directly due to the limitations of the retrospective study, so we chose "hospital days after surgery" to be an indirect measurement of the severity of any immediate complications. Also, we chose diabetes, body mass index (BMI), and hypertension to be the measurements of metabolic factors.

\section{Data and statistical analysis}

Data was collected retrospectively with medical record review. We reviewed patient data, including their demographic characteristics, symptoms, past medical histories, family medical histories, laboratory tests, liver function evaluations, operative findings, pathologic findings, treatment, and postoperative prognoses. Diabetic patients were defined as patients who had been previously diagnosed previously at any medical center, and as patients who had been diagnosed at a preoperative evaluation. In addition, hypertensive patients were defined as patients who had been previously diagnosed at any medical center, and as patients who had been diagnosed a preoperative evaluation. Major surgery was defined as the resection of three or more segments.

We used the American Joint Committee on Cancer (AJCC) $7^{\text {th }}$ edition for TNM staging, and differentiation and margin invasion were defined as pathological findings after surgery.

Statistical analysis were performed using SPSS software version 20.0. Continuous variables were compared using the Mann-Whitney $U$ test, and categorical variables with the $\chi^{2}$-test or Fisher's exact test as appropriate. Survival curves were generated using the Kaplan-Meier method and Cox regression analysis. All tests were two-tailed, and statistical significance was defined as $p<0.05$.

\section{RESULTS}

In total, 167 patients were involved. There were 133 males (79.6\%) and 34 females (20.3\%), with a mean age of $58.2 \pm 10.2$ years (range, 22-81 years). The mean BMI was $23.6 \pm 3.271 \mathrm{~kg} / \mathrm{m}^{2}$. There were 45 diabetic patients (26.9\%) and 122 non-diabetic patients (73.1\%). There were 25 hypertension patients (15.0\%) and 142 non-hy-

Table 1. Clinical characteristics of patients in the study

\begin{tabular}{|c|c|c|}
\hline Clinical data & Values & Percent \\
\hline \multicolumn{3}{|l|}{ Age (years) } \\
\hline Range & $22-81$ & \\
\hline Mean \pm SD & $58.2 \pm 10.2$ & \\
\hline \multicolumn{3}{|l|}{$\operatorname{Sex}(n)$} \\
\hline Males & 133 & 79.6 \\
\hline Females & 34 & 20.4 \\
\hline \multicolumn{3}{|l|}{ Body mass index } \\
\hline Mean \pm SD & $23.6 \pm 3.27$ & \\
\hline \multicolumn{3}{|l|}{ Diabetes (n) } \\
\hline Non-diabetes & 122 & 73.1 \\
\hline Diabetes & 45 & 26.9 \\
\hline \multicolumn{3}{|l|}{ Hypertension (n) } \\
\hline Non-hypertension & 142 & 85.0 \\
\hline Hypertension & 25 & 15.0 \\
\hline \multicolumn{3}{|l|}{ Hepatitis (n) } \\
\hline No hepatitis & 20 & 12.0 \\
\hline Hepatitis with HBV & 120 & 71.9 \\
\hline Hepatitis with HCV & 15 & 9.0 \\
\hline Hepatitis with HBV \& HCV & 3 & 1.8 \\
\hline Hepatitis with others & 9 & 5.4 \\
\hline \multicolumn{3}{|l|}{ MELD score (n) } \\
\hline $1-5$ & 31 & 18.6 \\
\hline $6-10$ & 130 & 77.8 \\
\hline $11-15$ & 4 & 2.4 \\
\hline $16-20$ & 2 & 1.2 \\
\hline
\end{tabular}

HBV, hepatitis B virus; HCV, hepatitis C virus; MELD, model for end-stage liver disease 
pertension patients $(85.0 \%)$ (Table 1).

There were 120 patients with HBV-associated hepatitis (71.9\%), 15 patients with HCV-related hepatitis (9.0\%), three patients with HBV- and HCV-related hepatitis $(1.8 \%), 9$ patients with other causes-related hepatitis $(5.4 \%)$, and 20 patients without hepatitis $(12.0 \%)$. There were 31 patients with a model for end-stage liver disease (MELD) score of 1-5 (18.6\%), 130 patients with a MELD score of 6-10 $(77.8 \%)$, four patients with a MELD score of 11-15 (2.4\%), and two patients with MELD score of 16-20 (1.2\%) (Table 1).

Major hepatic resection was performed in 49 patients (29.3\%) and minor hepatic resection was performed in 118 patients (70.7\%). Additionally, laparoscopic hepatic resection was performed in 11 patients $(6.6 \%)$ and open hepatic resection was performed in 156 patients (93.4\%). There were 29 patients who required perioperative transfusions $(17.4 \%)$ while 138 patients did not have perioperative transfusions $(82.6 \%)$. The mean hospital days after surgery were 16.2 \pm 24.7 days (range, 5-309 days) (Table 2).

The postoperative pathological examination reports of148 patients showed there were nine patients with Edmonson-Steiner grade I (6.1\%), 52 patients with grade II (35.1\%), 57 patients with grade III (38.5\%), and 30 patients with grade IV (20.3\%). The sizes of tumor varied from less than $5 \mathrm{~cm}$ in 119 patients $(71.3 \%)$ to more than $10 \mathrm{~cm}$ in 16 patients $(9.6 \%)$. There were nine patients with margin invasion (5.1\%) and one patient did not have a reported margin statues (Table 3).

The mean disease-free survival period was $23.2 \pm 27.7$ months, and the mean overall survival period was

Table 2. Perioperative surgical profiles

\begin{tabular}{lcc}
\hline \multicolumn{1}{c}{ Perioperative data } & Number & Percent \\
\hline Surgery & & \\
$\quad$ Major & 49 & 29.3 \\
$\quad$ Minor & 118 & 70.7 \\
Surgery type & 156 & 93.4 \\
$\quad$ Open & 11 & 6.6 \\
$\quad$ Laparoscopic & & \\
Perioperative transfusion & 138 & 82.6 \\
$\quad$ Non-transfusion & 29 & 17.4 \\
$\quad$ Transfusion & & \\
Hospital days after surgery & $5-309$ & \\
$\quad$ Range & $16.2 \pm 24.7$ & \\
$\quad$ Mean \pm SD & & \\
\hline
\end{tabular}

$34.8 \pm 33.1$ months. Nine of the 167 patients were margin-positive and one did not have a reported margin status. Recurrence was noted in 73 patients among the 157 during follow-up, and 56 patients among the 167 died. The 1-, 3-, and 5-year disease-free survival rates of the 157 patients were $70.0 \%, 56.6 \%$, and $54.1 \%$, respectively, and the respective overall survivalof the total patients were $91.6 \%, 79.0 \%$, and $73.0 \%$, respectively (Fig. 1).

We analyzed the effect of the metabolic factors such as diabetes, BMI, and hypertension on the prognosis of HCC patients undergoing hepatic resection. In this study, the influences of diabetes and BMI on disease-free and overall survival were not significant. However, hypertension was found to be a risk factor for disease-free and overall survival (Table 4).

In multivariate analysis, hypertension (HR 2.459, CI 1.359-4.449, $p=0.003$ ), perioperative transfusion (HR 1.923, CI 0.973-3.800, $p=0.060)$, major surgery (HR 3.023, CI 1.670-5.473, $p<0.001)$, resection with RFA or cryoablation (HR 7.351, CI 2.433-22.203, $p<0.001$ ), and margin invasion

Table 3. Postoperative pathological results

\begin{tabular}{|c|c|c|}
\hline Pathological data & Number & Percent \\
\hline Edmonson-Steiner grade & $(148)$ & \\
\hline I & 9 & 6.1 \\
\hline II & 52 & 35.1 \\
\hline III & 57 & 38.5 \\
\hline IV & 30 & 20.3 \\
\hline \multicolumn{3}{|l|}{ Tumor size } \\
\hline$<5 \mathrm{~cm}$ & 119 & 71.3 \\
\hline $5-10 \mathrm{~cm}$ & 32 & 19.1 \\
\hline$>10 \mathrm{~cm}$ & 16 & 9.6 \\
\hline \multicolumn{3}{|l|}{ Margin invasion } \\
\hline No & 157 & 94.0 \\
\hline Yes & 9 & 5.3 \\
\hline Not reported & 1 & 0.5 \\
\hline \multicolumn{3}{|l|}{$\mathrm{T}$ stage } \\
\hline 1 & 109 & 65.3 \\
\hline 2 & 34 & 20.4 \\
\hline 3 & 20 & 2.0 \\
\hline 4 & 4 & 2.4 \\
\hline \multicolumn{3}{|l|}{$\mathrm{N}$ stage } \\
\hline 0 & 166 & 99.4 \\
\hline 1 & 1 & 0.6 \\
\hline \multicolumn{3}{|l|}{ TNM stage } \\
\hline I & 108 & 64.7 \\
\hline II & 34 & 20.4 \\
\hline IIIa & 15 & 9.0 \\
\hline IIIb & 5 & 3.0 \\
\hline IIIc & 4 & 2.4 \\
\hline Iva & 1 & 0.6 \\
\hline
\end{tabular}


A

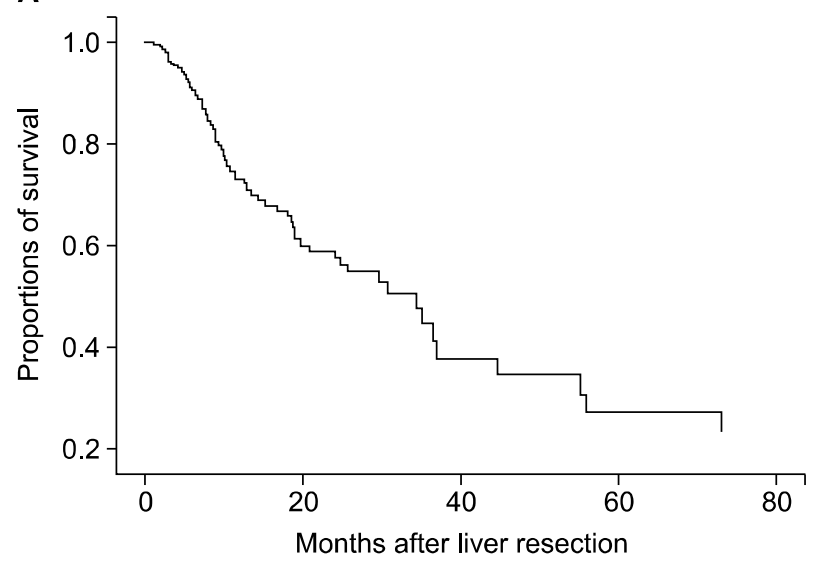

B

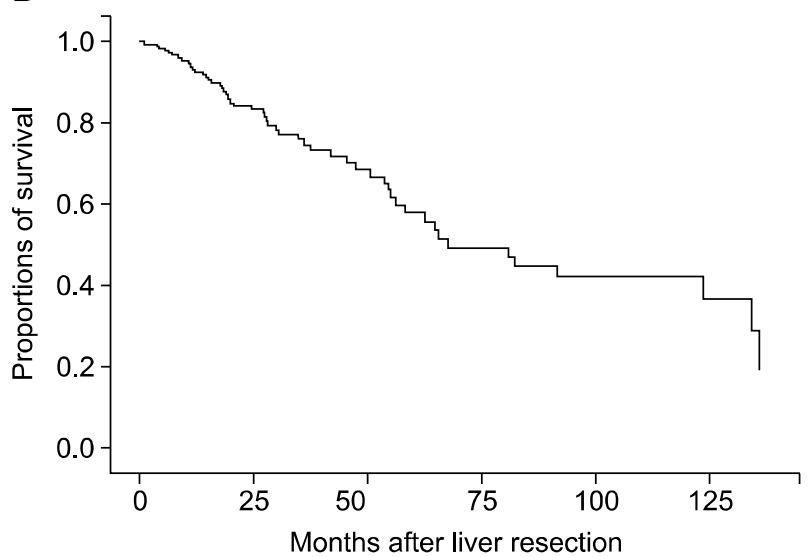

Fig. 1. Disease-free survival curve of 157 patients (A) and overall survival curve of all patients (B).

Table 4. Univariate analysis of metabolic factors predictive of overall survival and disease-free survival

\begin{tabular}{|c|c|c|c|c|c|c|c|c|}
\hline \multirow{3}{*}{$\begin{array}{c}\text { Parameter } \\
\text { Hypertension }\end{array}$} & \multicolumn{4}{|c|}{ Overall survival } & \multicolumn{4}{|c|}{ Disease-free survival } \\
\hline & \multirow{2}{*}{$\frac{\mathrm{HR}}{2.238}$} & \multicolumn{2}{|c|}{$95 \% \mathrm{CI}$} & \multirow{2}{*}{$\frac{p \text {-value }}{0.005}$} & \multirow{2}{*}{$\frac{\mathrm{HR}}{2.098}$} & \multicolumn{2}{|c|}{$95 \% \mathrm{CI}$} & \multirow{2}{*}{$\frac{p \text {-value }}{0.006}$} \\
\hline & & 1.268 & 3.951 & & & 1.243 & 3.542 & \\
\hline Diabetes & 1.371 & 0.764 & 2.460 & 0.290 & 1.261 & 0.769 & 2.070 & 0.358 \\
\hline BMI & 1.010 & 0.931 & 1.096 & 0.811 & 0.997 & 0.932 & 1.067 & 0.936 \\
\hline
\end{tabular}

HR, hazard ratio; CI, confidence interval; BMI, body mass index

Table 5. Multivariable analysis of prognostic factors that affect overall survival of HCC patients undergoing hepatic resection

\begin{tabular}{lcrr}
\hline \multicolumn{1}{c}{ Parameter } & HR & 95\% CI & $p$-value \\
\hline Hypertension & 2.459 & $1.359-4.449$ & 0.003 \\
Transfusion & 1.923 & $0.973-3.800$ & 0.060 \\
Major surgery & 3.023 & $1.670-5.473$ & $<0.001$ \\
Resection with RFA or cryoablation & 7.351 & $2.433-22.203$ & $<0.001$ \\
Margin & 4.346 & $1.750-10.791$ & 0.002 \\
\hline
\end{tabular}

HR, hazard ratio; CI, confidence intervals; RFA, radiofrequency ablation

Table 6. Multivariable analysis of prognostic factors that affected the disease-free survival of HCC patients undergoing hepatic resection

\begin{tabular}{lrrr}
\hline \multicolumn{1}{c}{ Parameter } & HR & 95\% CI & $p$-value \\
\hline Hypertension & 3.827 & $1.863-7.860$ & $<0.001$ \\
Albumin & 2.426 & $1.141-5.158$ & 0.021 \\
Resection with RFA or cryoablation & 10.014 & $3.206-31.275$ & $<0.001$ \\
Tumor size & 1.128 & $1.044-1.219$ & 0.002 \\
Transfusion & 2.361 & $1.162-4.797$ & 0.018 \\
\hline
\end{tabular}

HR, hazard ratio; CI, confidence intervals; RFA, radiofrequency ablation

(HR 4.346, CI 1.750-10.791, $p=0.002)$ were risk factors for overall survival (Table 5, Fig 2). Hypertension $(H R$ 3.827, CI 1.863-7.860, $p<0.001$ ), albumin (HR 2.426, CI 1.141-5.158, $p=0.021)$, resection with RFA or cryoablation
(HR 10.014, CI 3.206-31.275, $p<0.001)$, tumor size (HR 1.128 , CI 1.044-1.219, $p=0.002)$, and perioperative transfusion (HR 2.361, CI 1.162-4.797, $p=0.018)$ were all risk factors for disease-free survival (Table 6, Fig. 3). 


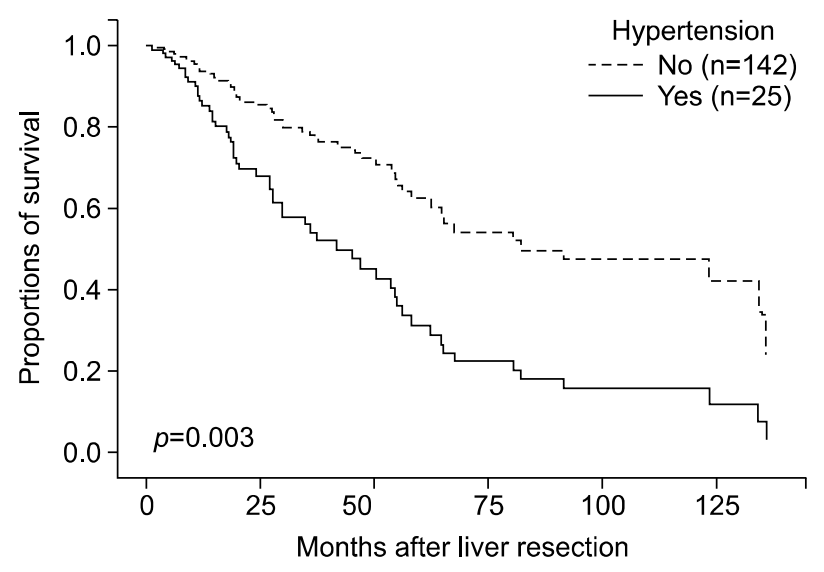

Fig. 2. Comparison of overall survival curves according to hypertension.

\section{DISCUSSION}

Metabolic syndrome is a constellation of problems that includes insulin resistance, obesity, dyslipidemia, and hypertension. ${ }^{2}$ In HCC, the relation with obesity appears to be mediated primarily by factors related to metabolic syndrome, nonalcoholic fatty liver disease, and nonalcoholic steatohepatitis. ${ }^{3}$ With this background, we studied the influence of certain metabolic factors, like diabetes, BMI, and hypertension on the prognosis of HCC patients undergoing hepatic resection.

Diabetes is considered a risk factor for the prognosis of HCC patients undergoing hepatic resection. ${ }^{9-12}$ Diabetes is not only developed as a complication of cirrhosis, but also is found in aggravated cirrhosis and HCC. ${ }^{13}$ However, other studies have shown that diabetes does not increase perioperative morbidity or mortality after resection of the liver in HCC, nor does it significantly influence long-term prognosis. ${ }^{14} \mathrm{We}$ investigated the relationship between diabetes and the prognosis of HCC patients undergoing hepatic resection, and found no statistical relationship; however, there were more diabetic patients in our HCC population than in the general population (45 patients among 167). This high incidence of diabetes in HCC patients was mainly related to their underlying liver cirrhosis. $^{13,15}$

In our study, hypertension was a risk factor of overall and disease-free survival in both univariate and multivariate analysis. Kaibori et al. ${ }^{4}$ suggest that hypertension is a risk factor for a poor prognoses after the resection of HCV-related HCC. Their study also showed there were

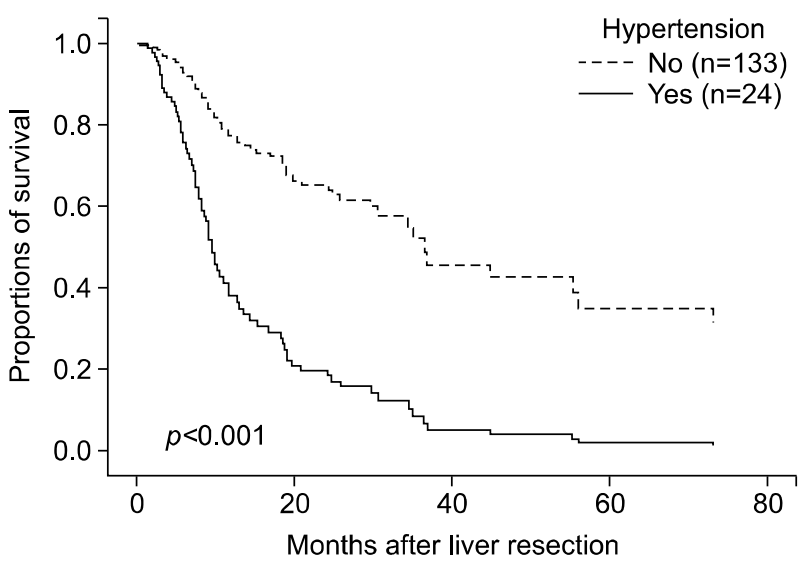

Fig. 3. Comparison of disease-free survival curves according to hypertension.

no significant differences between overall and disease-free survival with or without diabetes, and that a higher preoperative BMI was not associated with the outcome of surgical treatment. ${ }^{4}$ However, some studies have indicated that hypertension is a positive factor in the prognosis of patients with HCC. Hu et al. ${ }^{16}$ showed that baseline hypertension is associated with better overall survival in patients with HCC in two independent cohorts, and that the mechanism was associated with low vascular endothelial growth factor receptor 1 (VEGFR-1) expression levels in patients with HCC. Hence, the nature of the influence of hypertension on the prognosis of patients with HCC undergoing hepatic resection remains uncertain.

$\mathrm{HCC}$ is the most common primary cancer occurring in liver, and HBV is a main cause of HCC. In our study, viral hepatitis was not important to overall and disease-free survival. Although our results showed viral hepatitis were not related the prognosis of HCC patients with hepatic resection, Kubo et al. ${ }^{17}$ showed that hepatitis B status, tumor factors, and the type of operation affected cancer recurrence after surgery for HCC in patients with concurrent chronic HBV, as opposed to patients with previous chronic hepatitis $B$, in which only tumor factors affected the cancer recurrence rate. In addition, Ercolani et al. ${ }^{18}$ showed that HCV-positive patients with serum aspartate transaminase levels above twice the normal level have the highest risk for intrahepatic recurrence, and should be monitored carefully or offered alternative treatments.

Abdel-Wahab et al. ${ }^{7}$ showed that the factors that significantly predict survival were preoperative serum albumin, tumor differentiation, staging, tumor capsule, resection 
margin, vascular invasion, and operative blood transfusion, but that tumor differentiation was the only independent factor on multivariate analysis affecting long-term survival. Similarly, margin invasion affected overall in our study. Previous studies showed that perioperative transfusion was prognostic factor for HCC patients undergoing hepatic resection. ${ }^{19,20}$ Accordingly, our results also showed that perioperative transfusion affected overall and disease-free survival. In our study, tumor size affected disease-free survival. Nagao et al. ${ }^{21}$ showed that significant differences in survival patterns were noted when analyzed on the basis of preoperative alpha-fetoprotein level, tumor size, and tumor capsule.

Zarzavadjian et al. ${ }^{22}$ showed that metabolic syndrome, perioperative bleeding ( $\geq 1000 \mathrm{ml}$ ), middle hepatic vein resection, and primary hepatic malignancy were all related poor prognoses under a univariate analysis, and both middle hepatic vein resection and underlying primary hepatic malignancy were related to mortality under a multivariate analysis. Yeh et al. ${ }^{23}$ showed that the overall survival for HCC patients with liver cirrhosis who underwent hepatic resection could be stratified on the basis of the high value of alkaline phosphatase, tumor size, satellite lesions, and vascular invasion.

There were a few limitations of our study; a more detailed study of continuous variables like hospital days after surgery, alpha-fetoprotein level, and albumin level should be gathered, along with more evidence such as cut-off values. In addition, in the case of a large quantity of missing data regarding a specific factor, the factor was excluded from data; as such, we could not evaluate these factors.

In conclusion, hypertension, perioperative transfusion, and resection with RFA or cryoablation were identified as risk factors for both disease-free and overall survival after hepatic resection of HCC patients in our study. In terms of metabolic factors, diabetes and BMI were not associated with the prognosis of HCC patients undergoing hepatic resection, but hypertension was associated with disease-free and overall survival of HCC patients undergoing hepatic resection. However, these results remain controversial. Further research is needed to elucidate the influences of metabolic factors on the prognosis of patients with HCC, especially in the current era of increasing incidence of metabolic syndrome.

\section{REFERENCES}

1. Bruix J, Sherman M; American Association for the Study of Liver Diseases. Management of hepatocellular carcinoma: an update. Hepatology 2011;53:1020-1022.

2. Farrell GC, Larter CZ. Nonalcoholic fatty liver disease: from steatosis to cirrhosis. Hepatology 2006;43:S99-S112.

3. Siegel AB, Zhu AX. Metabolic syndrome and hepatocellular carcinoma: two growing epidemics with a potential link. Cancer 2009;115:5651-5661.

4. Kaibori M, Ishizaki M, Matsui K, Kitade H, Matsui Y, Kwon $\mathrm{AH}$. Evaluation of metabolic factors on the prognosis of patients undergoing resection of hepatocellular carcinoma. J Gastroenterol Hepatol 2011;26:536-543.

5. Shimada M, Matsumata T, Akazawa K, Kamakura T, Itasaka H, Sugimachi K, et al. Estimation of risk of major complications after hepatic resection. Am J Surg 1994;167:399-403.

6. Kim WW, Lee KW, Choi SH, Heo JS, Kim YI, Kim SJ, et al. Risk factors of morbidity and mortality following surgical resection for hepatocellular carcinoma. Korean J Hepatol 2004;10: 51-61.

7. Abdel-Wahab M, El-Husseiny TS, El Hanafy E, El Shobary M, Hamdy E. Prognostic factors affecting survival and recurrence after hepatic resection for hepatocellular carcinoma in cirrhotic liver. Langenbecks Arch Surg 2010;395:625-632.

8. Shimada M, Takenaka K, Fujiwara Y, Gion T, Shirabe K, Yanaga $\mathrm{K}$, et al. Risk factors linked to postoperative morbidity in patients with hepatocellular carcinoma. Br J Surg 1998;85: 195-198.

9. Teoh NC, Fan JG. Diabetes mellitus and prognosis after curative therapy for hepatocellular carcinoma: alas, still grave for those who are hyperglycemic. J Gastroenterol Hepatol 2008;23:16331634.

10. Huo TI, Lui WY, Huang YH, Chau GY, Wu JC, Lee PC, et al. Diabetes mellitus is a risk factor for hepatic decompensation in patients with hepatocellular carcinoma undergoing resection: a longitudinal study. Am J Gastroenterol 2003;98:2293-2298.

11. Yanaga K, Matsumata T, Hayashi H, Shimada M, Urata K, Suehiro T, et al. Effect of diabetes mellitus on hepatic resection. Arch Surg 1993;128:445-448.

12. Ikeda Y, Shimada M, Hasegawa H, Gion T, Kajiyama K, Shirabe K, et al. Prognosis of hepatocellular carcinoma with diabetes mellitus after hepatic resection. Hepatology 1998;27:15671571.

13. García-Compean D, Jaquez-Quintana JO, Maldonado-Garza H. Hepatogenous diabetes. Current views of an ancient problem. Ann Hepatol 2009;8:13-20.

14. Poon RT, Fan ST, Wong J. Does diabetes mellitus influence the perioperative outcome or long term prognosis after resection of hepatocellular carcinoma? Am J Gastroenterol 2002;97:14801488 .

15. Kaczynski J, Hansson G, Wallerstedt S. Diabetes: one of few remarkable differences in clinicopathologic features between cirrhotic and noncirrhotic Swedes with hepatocellular carcinoma. Dig Dis Sci 2006;51:796-802.

16. Hu YF, Chen TC, Chau GY, Yang TL, Liu CJ, Chen MH, et al. Baseline hypertension: new insight into the potential predictors of survival in patients with hepatocellular carcinoma. Int J Cardiol 2013;168:2979-2981.

17. Kubo S, Tanaka $H$, Shuto $T$, Takemura S, Yamamoto $T$, Kanazawa A, et al. Clinicopathologic features and outcome after liver resection for hepatocellular carcinoma in patients with concurrent versus previous chronic hepatitis B. Surg Today 2005;35:216-222. 
18. Ercolani G, Grazi GL, Ravaioli M, Del Gaudio M, Gardini A, Cescon M, et al. Liver resection for hepatocellular carcinoma on cirrhosis: univariate and multivariate analysis of risk factors for intrahepatic recurrence. Ann Surg 2003;237:536-543.

19. Asahara T, Katayama K, Itamoto T, Yano M, Hino H, Okamoto $\mathrm{Y}$, et al. Perioperative blood transfusion as a prognostic indicator in patients with hepatocellular carcinoma. World J Surg 1999;23: 676-680.

20. Poon RT, Fan ST, Lo CM, Ng IO, Liu CL, Lam CM, et al. Improving survival results after resection of hepatocellular carcinoma: a prospective study of 377 patients over 10 years. Ann Surg 2001;234:63-70.
21. Nagao T, Inoue S, Goto S, Mizuta T, Omori Y, Kawano N, et al. Hepatic resection for hepatocellular carcinoma. Clinical features and long-term prognosis. Ann Surg 1987;205:33-40.

22. Zarzavadjian Le Bian A, Costi R, Constantinides V, Smadja C. Metabolic disorders, non-alcoholic fatty liver disease and major liver resection: an underestimated perioperative risk. J Gastrointest Surg 2012;16:2247-2255.

23. Yeh CN, Chen MF, Lee WC, Jeng LB. Prognostic factors of hepatic resection for hepatocellular carcinoma with cirrhosis: univariate and multivariate analysis. J Surg Oncol 2002;81:195202. 